\title{
Article \\ Clonal Expansion of Tumor-Infiltrating T Cells and Analysis of the Tumor Microenvironment within Esophageal Squamous Cell Carcinoma Relapsed after Definitive Chemoradiation Therapy
}

\author{
Takahiro Mori 1,2,3,*(D), Kenichi Kumagai ${ }^{2,4,5}$, Keisuke Nasu ${ }^{2,5}$, Takamasa Yoshizawa ${ }^{2,5}$, Koji Kuwano ${ }^{2,6}$, \\ Yoshiki Hamada ${ }^{5}$, Hideki Kanazawa ${ }^{6}$ and Ryuji Suzuki ${ }^{2}$
}

check for

updates

Citation: Mori, T.; Kumagai, K.;

Nasu, K.; Yoshizawa, T.; Kuwano, K.;

Hamada, Y.; Kanazawa, H.; Suzuki, R.

Clonal Expansion of

Tumor-Infiltrating T Cells and

Analysis of the Tumor

Microenvironment within Esophageal Squamous Cell Carcinoma Relapsed after Definitive Chemoradiation Therapy. Int. J. Mol. Sci. 2021, 22, 1098. https://doi.org/10.3390/ ijms22031098

Academic Editor: Lukas J. A.

C. Hawinkels

Received: 24 December 2020

Accepted: 19 January 2021

Published: 22 January 2021

Publisher's Note: MDPI stays neutral with regard to jurisdictional claims in published maps and institutional affiliations.

Copyright: (c) 2021 by the authors. Licensee MDPI, Basel, Switzerland. This article is an open access article distributed under the terms and conditions of the Creative Commons Attribution (CC BY) license (https:// creativecommons.org/licenses/by/ $4.0 /)$.
1 Departments of Clinical Oncology and Gastroenterological Surgery, National Hospital Organization Sagamihara National Hospital, 18-1 Sakuradai, Minami-ku, Sagamihara 252-0392, Japan

2 Department of Rheumatology and Clinical Immunology, Clinical Research Center for Rheumatology and Allergy, National Hospital Organization Sagamihara National Hospital, 18-1 Sakuradai, Minami-ku, Sagamihara 252-0385, Japan; kumagaik-ora@h.u-tokyo.ac.jp (K.K.); pd19008@stu.tsurumi-u.ac.jp (K.N.); pd19010@stu.tsurumi-u.ac.jp (T.Y.); kuwano.koji.gq@mail.hosp.go.jp (K.K.); suzuki@repertoire.co.jp (R.S.)

3 Tohoku University Graduate School of Medicine, 1-1 Seiryo-machi, Aobaku, Sendai 980-8574, Japan

4 Department of Oral-Maxillofacial Surgery and Orthodontics, The University of Tokyo Hospital, 7-3-1 Hongo, Bunkyo-ku, Tokyo 113-8655, Japan

5 Department of Oral and Maxillofacial Surgery, School of Dental Medicine, Tsurumi University, 2-1-3 Tsurumi, Tsurumi-ku, Yokohama 230-8501, Japan; hamada-y@tsurumi-u.ac.jp

6 Department of Surgery, National Hospital Organization Sagamihara National Hospital, 18-1 Sakuradai, Minami-ku, Sagamihara 252-0392, Japan; kanazawa.hideki.ma@mail.hosp.go.jp

* Correspondence: westendc7m@gmail.com

Abstract: (1) Background: Comparable prognoses after definitive chemoradiation therapy (CRT) to surgery alone for esophageal squamous cell carcinoma (ESCC) have been previously reported; however, no robust prognostic markers have been established. The clonality of tumor-infiltrating lymphocytes (TILs) and tumor microenvironments (TMEs) in ESCC relapsed after CRT were examined to explore prognostic markers. (2) Methods: Clonality of TIL and TME were examined in ESCC with and without preceding CRT, as well as oral squamous cell carcinoma (OSCC) and healthy volunteers as controls. The clonality of TIL was assessed by T-cell receptor (TCR) $\alpha$ and $\beta$ repertoire analyses and evaluated by diversity indices. The TME was assessed by quantitative polymerase chain reaction evaluating $P D-L 1$ and CD8B. (3) Results: The clonal expansion of TIL was significantly induced within ESCCs and OSCCs, when compared to healthy volunteers, and was mostly induced within ESCCs after definitive CRT. Diversity indices of TIL were not associated with the prognosis, but the ratio of $P D-L 1$ mRNA to $C D 8 B$ mRNA in TME was significantly associated with a poor prognosis after salvage surgery $(p=0.007)$. (4) Conclusions: The clonal expansion of TIL is induced after definitive CRT for ESCC, and the ratio of PD-L1 mRNA to CD8B mRNA within tumor tissues is a prognostic marker candidate for salvage esophagectomy after CRT.

Keywords: esophageal squamous cell carcinoma; definitive chemoradiation therapy; tumor-infiltrating T cell; TCR repertoire; PD-L1; CD8; salvage esophagectomy; prognostic marker

\section{Introduction}

Esophageal squamous cell carcinoma (ESCC) is one of the most lethal neoplasms in both Japan and other parts of the world [1-3]. Multimodal medical treatments consisting of neoadjuvant chemotherapy or chemoradiation therapy followed by surgery is the current gold standard of treatment, especially for patients with advanced stage II or III ESCCs [4-6]. Alternatively, definitive chemoradiation therapy (CRT) itself may be another powerful treatment for carcinoma curability, because some cases have been reported to achieve 
pathologically complete remission (pCR) after neoadjuvant CRT [7]. Comparable prognoses after definitive CRT to surgery alone have been reported by a couple of independent prospective studies [8,9]. Nevertheless, not all patients with ESCC have been treated with definitive CRT as a first-line treatment. One of the reasons to refrain from definitive CRT as a first-line treatment option is that local relapses may then require salvage esophagectomies, which may have a high risk for severe comorbidities such as pulmonary complications [10]. Hence, it is necessary to treat them using the optimal medical procedures that have been determined by robust predictive or prognostic markers. Various studies have been performed to identify the predictive or prognostic factors of medical procedures for ESCC, including CRT, although no robust marker has yet been established [11-13].

Recent advances in molecular biology and tumor immunology have revealed the fact that the therapeutic effects of chemotherapy or radiation should be greatly influenced by the tumor immunologic microenvironment [14-17]. Actually, many studies have suggested that the factors relating to the tumor microenvironment (TME), such as PD-L1 expression or the presence of tumor-infiltrating lymphocytes (TIL), could be prognostic or predictive marker candidates for chemotherapy or chemoradiation in various types of human cancers, including ESCC [18-23]. These previous reports have encouraged us to examine and characterize the specific features of TIL and TME, especially within ESCCs that have relapsed after definitive CRT, to explore the prognostic maker candidates of definitive CRT followed by salvage esophagectomy. However, almost all the patients with advanced ESCCs are treated with neoadjuvant CRT or chemotherapy before surgery in Japan $[24,25]$. This suggests the difficulty of enrolling a sufficient number of patients with advanced ESCCs that have been solely treated by surgery as a control group. Instead, we enrolled patients with oral squamous cell carcinoma (OSCC) in this study; OSCC is considered to be comparable to ESCC, because they share similar pathologic diagnoses, squamous cell carcinoma, carcinogenic pathways (such as alcohol drinking and smoking), and molecular features [26-29]. Additionally, ESCC is usually treated by neoadjuvant CRT or chemotherapy followed by surgery, as described above, whereas OSCC is rarely treated with neoadjuvant chemotherapy or CRT before surgery.

In this study, we reveal the results of diversity analyses of the T-cell receptor (TCR) repertoire of TIL within post-definitive CRT locally relapsed ESCC by comparing them to ESCC and OSCC that have both been treated only by surgery in order to reveal the specific immunological features initiated by definitive CRT. We also demonstrate the molecular features of the TME, especially in ESCCs relapsed after definitive CRT, which could be prognostic marker candidates after salvage esophagectomy.

\section{Results}

2.1. Clonal Expansion of Tumor-Infiltrating T Lymphocytes Was Significantly Induced within Esophageal and Oral Squamous Cell Carcinoma Tumor Tissues and Mostly Induced within Esophageal Squamous Cell Carcinoma That Relapsed after Definitive CRT

The results of the repertoire analyses of complementarily determining region three of $T C R \alpha(T R A)$ or $\beta(T R B)$ are shown in three dimensional graphs in Figure 1. The diversity and evenness of the TCR repertoire are defined as the Shannon-Weaver index $\mathrm{H}$, inverse Simpson's index $(1 / \lambda)$, and Pielou's evenness in Table 1 or Supplementary Table S1. Based on the results of the Shapiro-Wilk $W$ test and Levene's test, the mean value of each clinical group was statistically compared to an unpaired Student's $t$-test, Welch's $t$-test, or MannWhitney U test, as described below (Figure 2).

The obtained results indicated that the divergency and evenness of the TCR repertoire in healthy volunteers were always significantly higher than in patients with OSCC $(p<0.001$ in the Shannon-Weaver Index H for TRA, $p<0.001$ in Pielou's evenness for TRA, $p<0.001$ in inverse Simpson $(1 / \lambda)$ for $T R A, p<0.001$ in the Shannon-Weaver Index $H$ for $T R B$, $p<0.001$ in Pielou's evenness for TRB, and $p=0.002$ in inverse Simpson $(1 / \lambda)$ for TRB); in patients with ESCC treated by surgery alone $(p<0.001$ in the Shannon-Weaver Index $\mathrm{H}$ for TRA, $p<0.001$ in Pielou's evenness for TRA, and $p=0.001$ in the Shannon-Weaver Index $\mathrm{H}$ for TRB); or in patients with ESCC that relapsed after definitive CRT $(p<0.001$ in 
the Shannon-Weaver Index H for TRA, $p<0.001$ in Pielou's evenness for TRA, $p<0.001$ in the Shannon-Weaver Index H for TRB, and $p<0.001$ in Pielou's evenness for TRB), except, in some cases, as follows: inverse Simpson $(1 / \lambda)$ for TRA either in patients with ESCC surgery alone $(p=0.444)$ or with ESCC recurrence after definitive CRT $(p=0.182)$, inverse Simpson $(1 / \lambda)$ for TRB either in patients with ESCC surgery alone $(p=0.491)$ or with ESCC recurrence after definitive CRT $(p=0.236)$, and Pielou's evenness for TRB for patients with ESCC surgery alone $(p=0.236)$ (Figure 2$)$. It should be noted that the results in healthy volunteers were based on the different materials, such as peripheral white blood cells.
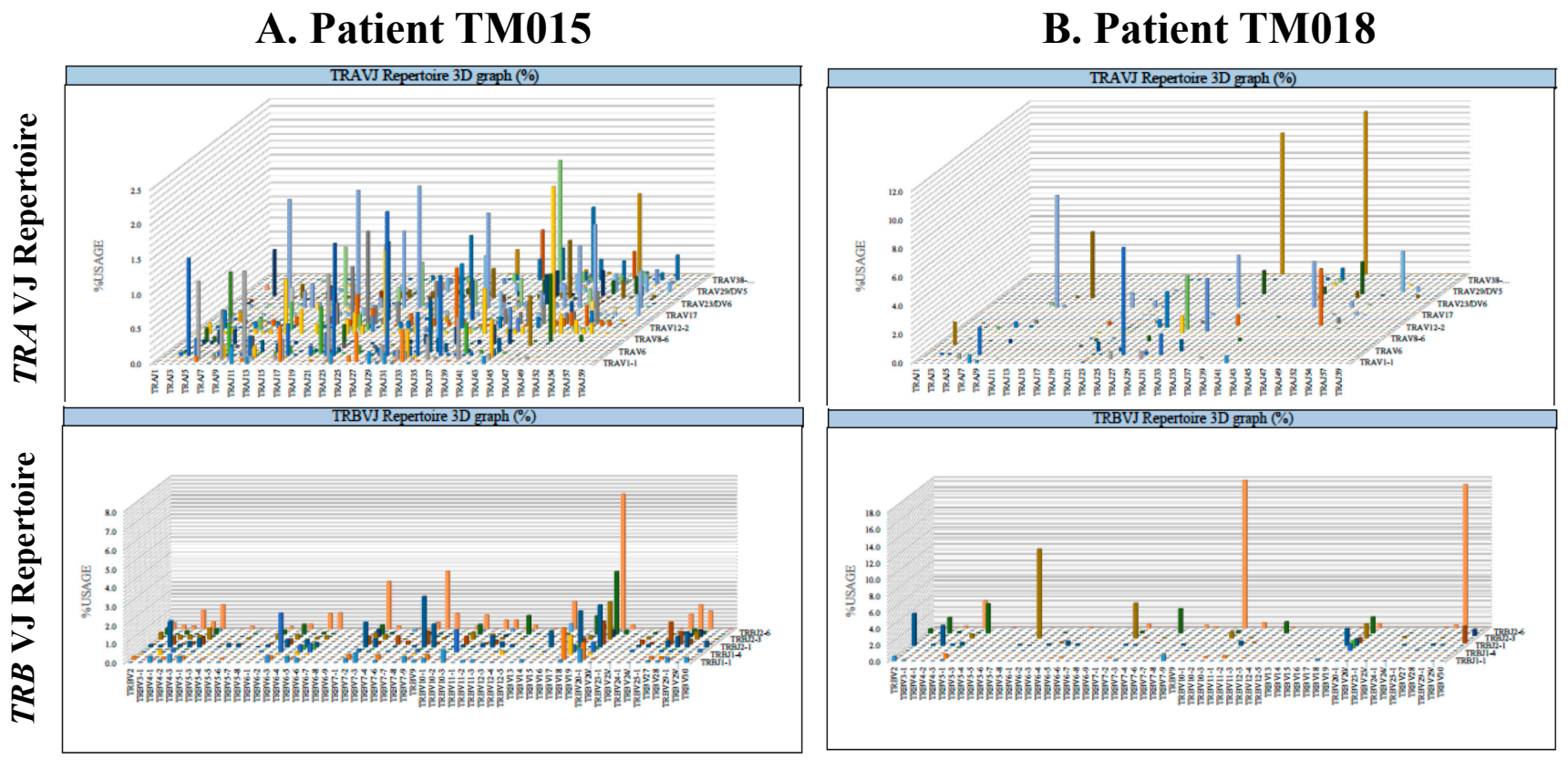

Figure 1. The typical results of the T-cell receptor $\alpha(T R A)$ and TCR $B(T R B)$ repertoires, each of esophageal squamous cell carcinoma (ESCC) treated by surgery only (A) and ESCC relapsed after definitive chemoradiation therapy (CRT) (B). The $T R A$ repertoire and TRB repertoire are shown in the upper and lower panels, respectively. Three-dimensional graphs indicate the combination of using TRAJ on the $\mathrm{x}$-axis and TRAV on the y-axis or of TRBV on the $\mathrm{x}$-axis and TRBJ on the $\mathrm{y}$-axis, with the frequency (percentage) of each clone on the z-axis. Oligoclonal expansion is observed more in ESCC relapsed after definitive CRT (B) than in ESCC treated by surgery alone (A).

As for OSCCs and ESCCs, RNAs were extracted from tumor tissues that were obtained by surgical resection and used for TCR sequencing. No statistically significant differences were observed between patients with OSCC and ESCC; both treated by surgery alone ( $p=0.288$ in the Shannon-Weaver index $\mathrm{H}$ for TRA, $p=0.582$ in Pielou's evenness for $T R A, p=0.800$ in inverse Simpson $(1 / \lambda)$ for $T R A, p=0.501$ in the Shannon-Weaver index $\mathrm{H}$ for $T R B, p=0.538$ in Pielou's evenness for $T R B$, and $p=0.800$ in inverse Simpson $(1 / \lambda)$ for $T R B)$ and the mean value of each diversity index was similar between these two groups (Figure 2). On the other hand, statistically significant differences were observed between the OSCC group and ESCC that relapsed after definitive CRT $(p<0.001$ in the Shannon-Weaver index $\mathrm{H}$ for TRA, $p<0.001$ in Pielou's evenness for TRA, $p<0.001$ in the Shannon-Weaver index H for TRB, $p<0.001$ in Pielou's evenness for TRB, and $p=0.014$ in inverse Simpson $(1 / \lambda)$ for TRB), except for inverse Simpson $(1 / \lambda)$ for TRA $(p=0.387)$ (Figure 2). Between the ESCC treated by surgery alone group and ESCC relapsed after definitive CRT group, the differences were not statistically significant, although the mean diversity indices were always higher in those of surgery alone than in those that relapsed after definitive CRT. This was likely due to the limited number of patients with ESCC treated by surgery alone $(p=0.252$ in the Shannon-Weaver index $\mathrm{H}$ for TRA, $p=0.171$ in Pielou's evenness for TRA, $p=0.418$ for inverse Simpson $(1 / \lambda)$ for TRA, $p=0.518$ in 
the Shannon-Weaver index $\mathrm{H}$ for $T R B, p=0.525$ for inverse Simpson $(1 / \lambda)$ for $T R B$, and $p=0.460$ in Pielou's evenness for TRB).

Table 1. The clinical and pathologic data of patients with esophageal squamous cell carcinoma (ESCC) or with oral squamous cell carcinoma (OSCC) examined in this study. CRT: chemoradiation therapy.

\begin{tabular}{|c|c|c|c|c|c|c|c|}
\hline \multirow[b]{2}{*}{ Disease } & \multirow[b]{2}{*}{$\begin{array}{l}\text { First-Line } \\
\text { Therapy }\end{array}$} & \multirow[b]{2}{*}{$\begin{array}{l}\text { Radiation } \\
\text { Dose (gy) }\end{array}$} & \multirow[b]{2}{*}{$\begin{array}{l}\text { Concurrent } \\
\text { Chemotherapy }\end{array}$} & \multirow[b]{2}{*}{ fTNM } & \multicolumn{3}{|c|}{ Prognosis After Salvage Esophagectomy } \\
\hline & & & & & $\begin{array}{c}\text { Cancer } \\
\text { Recurrence }\end{array}$ & Prognosis & $\begin{array}{c}\text { Recurrence-Free } \\
\text { Survival } \\
\text { (Months) }\end{array}$ \\
\hline ESCC & CRT & 60 & $\mathrm{CDDP}+5-\mathrm{FU}$ & T2N1M0 & yes & cancer death & 18.4 \\
\hline ESCC & CRT & 60 & CDDP+5-FU & T2N1M0 & no & survive & 22.9 \\
\hline ESCC & CRT & 60 & $\mathrm{CDDP}+5-\mathrm{FU}$ & T3NOM0 & no & survive & 74.1 \\
\hline ESCC & CRT & 60 & $\mathrm{CDDP}+5-\mathrm{FU}$ & T4N1M0 & yes & cancer death & 6.6 \\
\hline ESCC & surgery alone & 0 & no & T2N1M0 & no & survive & 117.7 \\
\hline ESCC & surgery alone & 0 & no & T3N0M0 & no & died from other disease & 67.2 \\
\hline ESCC & CRT & 60 & $\mathrm{CDDP}+5-\mathrm{FU}$ & T3N0M0 & yes & cancer death & 9.5 \\
\hline OSCC & surgery alone & 0 & no & T2N0M0 & & & \\
\hline OSCC & surgery alone & 0 & no & T1N1M0 & & & \\
\hline OSCC & surgery alone & 0 & no & $\mathrm{T} 2 \mathrm{~N} 2 \mathrm{bM} 0$ & & & \\
\hline OSCC & surgery alone & 0 & no & T2N1M0 & & & \\
\hline OSCC & surgery alone & 0 & no & T4aNOM0 & & & \\
\hline OSCC & surgery alone & 0 & no & T4aN3bM1 & & & \\
\hline OSCC & surgery alone & 0 & no & T1N1M0 & & & \\
\hline OSCC & surgery alone & 0 & no & T4N1M0 & & & \\
\hline OSCC & surgery alone & 0 & no & T1N1M0 & & & \\
\hline OSCC & surgery alone & 0 & no & T3N0M0 & & & \\
\hline OSCC & surgery alone & 0 & no & T1N1M0 & & & \\
\hline OSCC & surgery alone & 0 & no & $\mathrm{T} 2 \mathrm{~N} 2 \mathrm{bM} 0$ & & & \\
\hline OSCC & surgery alone & 0 & no & T3N0M0 & & & \\
\hline
\end{tabular}
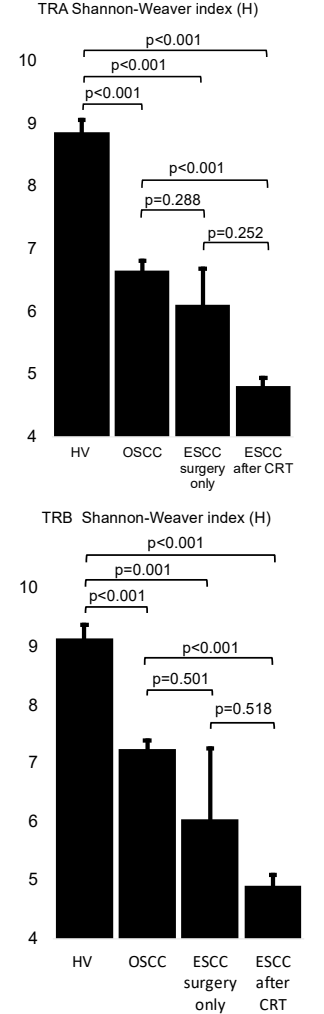

Figure 2. T-cell receptor (TCR) diversity indices in healthy volunteers (HV), oral squamous cell carcinoma (OSCC) treated by surgery alone, and esophageal squamous cell carcinoma (ESCC) with or without preceding chemoradiation therapy. Each bar indicates a mean value of each TCR repertoire index with an error bar as the standard error. 
2.2. Divergency of the T-Cell Receptor Repertoire of Tumor-Infiltrating Lymphocytes Was Not Significantly Different between Patients with Good or Poor Prognoses after Salvage Surgery for Esophageal Squamous Cell Carcinoma Locally Relapsed after Definitive Chemoradiation Therapy

The results of the divergency evaluation for the TCR repertoire of TIL for locally relapsed ESCCs after definitive CRT are shown in Figure 3A,B. The divergency was not statistically different between patients with good prognoses and those with poor prognoses ( $p=0.765$ for the Shannon-Weaver index $\mathrm{H}, p=0.525$ for inverse Simpson $(1 / \lambda)$, and $p=0.958$ for Pielou's evenness for TRA and $p=0.599$ for the Shannon-Weaver Index $\mathrm{H}$, $p=0.631$ for inverse Simpson $(1 / \lambda)$, and $p=0.668$ for Pielou's evenness for TRB).

A. Patient TM001

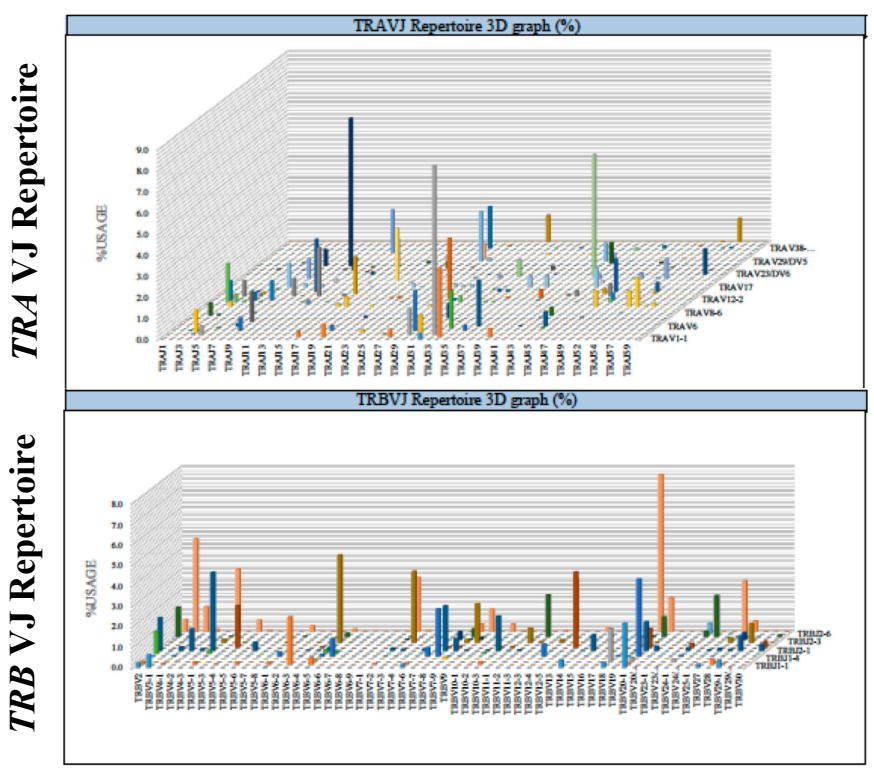

B. Patient TM003

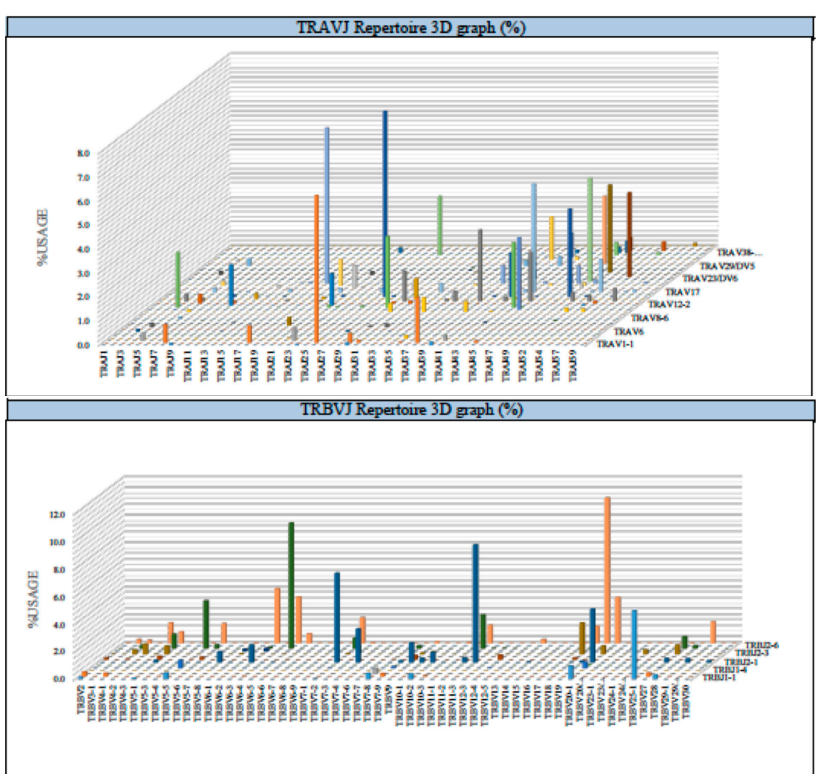

Figure 3. The typical results of the TRA and TRB repertoires in esophageal squamous cell carcinomas (ESCCs)

2.3. Ratio of PD-L1 $m R N A$ to CD8B $m R N A$ within Tumor Tissues Significantly Higher in Patients with Poor Prognoses after Salvage Surgery for Locally Relapsed Esophageal Squamous Cell Carcinoma after Definitive Chemoradiation Therapy

The results of the quantitative PCR (qPCR) analyses are shown in Figure 4. The ratio of $P D$ - $L 1$ mRNA to CD8B mRNA was statistically different between patients with good prognoses and with poor prognoses after the salvage surgeries for ESCCs relapsed after definitive CRT ( $p=0.007)$, although the expression levels of $P D-L 1$ and $C D 8 B$, adjusted by GAPDH mRNA, were not statistically different between these two groups $(p=0.311$ and $p=0.490$, respectively). 

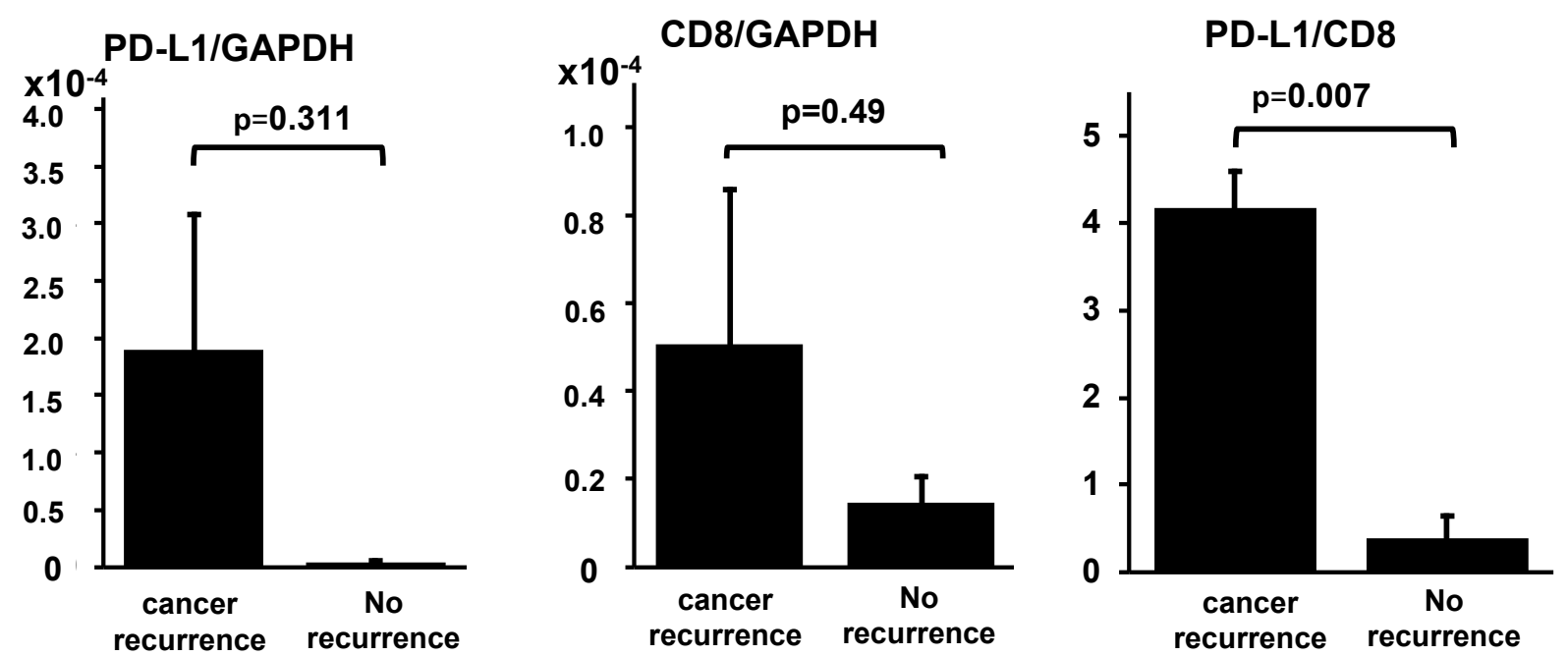

Figure 4. The results of tumor microenvironment analyses by quantitative polymerase chain reaction in both the cancer recurrence group and no recurrence group after salvage esophagectomy for local failure of definitive CRT. Each bar indicates a mean value with an error bar as the standard error. The ratio of PD-L1 mRNA to CD $8 B$ mRNA was statistically different between patients with good prognoses and with poor prognoses after the salvage surgeries $(p=0.007)$. However, each expression level of $P D-L 1$ and $C D 8 B$, adjusted by GAPDH mRNA, was not statistically different between these two groups.

\section{Discussion}

To our knowledge, this is the first report to analyze the clonality of the TIL and TMEs within ESCCs that relapsed after definitive CRT. Our results suggest that clonal expansion of the TIL was induced after definitive CRT; moreover, the ratio of $P D-L 1$ mRNA to CD8 mRNA could be used as a prognostic marker candidate for salvage surgery after a local recurrence after definitive CRT.

In particular, our results showed that the oligoclonality of TIL was statistically induced in OSCC and ESCC with or without preceding CRT, when compared to the peripheral blood in healthy volunteers (Figure 2). This suggests that the clonal expansion of TIL could be induced by the stimulation of neoantigens derived from tumor cells through the process consisting of cancer antigen presentation, the priming and trafficking of $\mathrm{T}$ cells, and infiltration of T cells into tumors, which is commonly known as "the cancer-immunity cycle" reviewed by Chen and Mellman [30]. We also investigated whether the oligoclonality of TIL was more significantly induced after definitive CRT, because radiation and chemotherapy have been previously reported to induce neoantigens by disrupting cancer cells $[17,31,32]$. Preferably, we could compare the clonality of TIL between stage-adjusted ESCCs with and without preceding definitive CRT directly; however, it is difficult to recruit patients with stage II or III ESCCs that did not have preceding neoadjuvant therapies in Japan. This is because neoadjuvant chemotherapies or chemoradiation therapies are recommended before esophagectomy, which is supported by robust data from clinical studies [24,25]. Only two patients with stage II or stage III ESCCs that did not have preceding neoadjuvant therapies were enrolled in this study; accordingly, we predicted that the number of enrolled patients might not be enough to meet a statistical significance. Instead, we enrolled patients with stage II or III OSCCs in the study, because they likely had similar TMEs to ESCCs, and they shared the same pathologic diagnoses and carcinogenic pathways as described above [26-28]. Besides, patients with stage II or III OSCCs are usually treated by surgery alone, without any preceding neoadjuvant therapies. In fact, 13 patients with OSCCs that were treated by surgery alone were enrolled in this study (Table 1). As expected, diversity analyses of the TCR repertoire showed quite similar results in each diversity index between the OSCC and ESCC both treated by surgery alone (Figure 2), while significantly stronger oligoclonality in ESCC after definitive CRT than in OSCC was certainly observed $(p<0.001$ in the Shannon-Weaver index $\mathrm{H}$ for TRA, $p<0.001$ in Pielou's evenness for TRA, $p<0.001$ 
in the Shannon-Weaver index $\mathrm{H}$ for TRB, $p<0.001$ in Pielou's evenness for TRB, and $p=0.014$ in inverse Simpson $(1 / \lambda)$ for TRB). Taken together, it might be plausible that the oligoclonality of TIL in ESCC after CRT might be more significantly induced than in ESCC treated by surgery alone. This clonal expansion of TIL induced by CRT is consistent with a previous study showing CD8+ TIL induction in ESCC after neoadjuvant chemotherapy, as evaluated by CD8 immunohistochemistry [33].

Next, we compared the diversity of the TCR repertoire between patients with good or poor prognoses after salvage surgery for ESCC that locally relapsed after definitive CRT. From previous reports, we predicted that patients with a good prognosis might contain a stronger clonal expansion of TIL, which might play an important role in improving the prognosis after definitive CRT [32,34]. As for TIL induction within tumors, the presence of CD8+ T-cell infiltration was previously reported to be a favorable prognostic marker in ESCC treated by surgery [35]. Moreover, higher post-CRT CD8+ TIL density was reportedly a favorable prognostic indicator after concurrent chemoradiation therapy for lung cancers [21]. Tumor remission and TILs during CRT were previously suggested to be predictive markers for pathological CR (pCR) in ESCC after CRT [23]. However, the results obtained in this study indicated no statistical differences between these two groups (Figure $3 \mathrm{~A}, \mathrm{~B}$ ). It could be possible that ESCCs remaining in pCR after definitive CRT could have a stronger expansion of clonality of TIL than the locally relapsed cases that were examined in this study. However, it is quite difficult to analyze the clonality of TIL by the TCR repertoire diversity only from the endoscopic biopsies, and physicians or surgeons usually watch carefully the patients with ESCCs after CRT without surgical treatments as far as they remain in clinical CR. The salvage surgery is only required at a local relapse after CRT. Therefore, our results just suggest that the clonal expansion of TIL is significantly induced after CRT, which is not statistically associated with the prognosis after salvage esophagectomy in cases with relapsed ESCCs, nor in cases remaining in pCR.

We then analyzed the TME to find the key factors separating the two groups, with a good prognosis and with a poor prognosis, after salvage surgeries. In the qPCR assay, we observed that the ratio of $P D-L 1$ mRNA to CD8B mRNA was statistically higher in those with a poor prognosis $(p=0.007)$, although both $P D-L 1$ and $C D 8 B$, adjusted by $G A P D H$, were not statistically different (Figure 4). These results prompted us to hypothesize that the clonal expansion of TIL, which occurred in all the cases after definitive CRT examined in this study, might not be enough and should be followed or accompanied by PD-L1 suppression, although the upregulation of PD-L1 is likely to be induced after neoadjuvant chemotherapy, as previously reported [33]. This hypothesis is plausible, because similar results have been reported in various human cancers; the lack of PDL1 expression and the combined presence of CD8+ TIL were associated with favorable survival rates in stage III non-small cell lung carcinoma after CRT, as well as in patients with extrahepatic bile duct cancer after surgery and adjuvant chemoradiation therapy [18,22,36]; the immunophenotypes determined by TIL density and their spatial organization in the tumor, which might be influenced by the infiltration of CD8+ TIL and expression of PD-L1 or PD-1, were reportedly prognostic marker candidates of tongue squamous cell carcinoma [37]. The salvage surgery for ESCC that locally relapses after definitive CRT is surgically feasible; however, severe comorbidities such as pneumonia or anastomotic leakage are often reported $[7,38]$. Therefore, it is necessary to treat them with the optimal medical treatments; from this study, salvage surgery should be considered for ESCC with a low ratio of PD-L1/CD8 after definitive CRT, while a PD-1/PD-L1 blockade should be recommended for those with a high ratio of PD-L1/CD8 [30]. However, the results obtained in this study are based on the surgical specimens, not on the endoscopic biopsies or peripheral bloods. In order to establish the predictive marker, the results should be validated by another cohort by using endoscopic biopsies before the initiation of each treatment-in particular, the ratios of PD-L1/CD8 evaluated on the endoscopic biopsies before salvage esophagectomy. 


\section{Materials and Methods}

\subsection{Patients}

The clinical and pathologic data of the enrolled patients are summarized in Table 1. All the tumors of seven ESCCs and 13 OSCCs were diagnosed as squamous cell carcinomas by board-certified pathologists. Among the seven ESCCs, two patients were treated only by surgery, and the others were primarily treated by definitive CRT, followed by salvage esophagectomy. Among the five patients treated by salvage esophagectomy, two survived without cancer recurrence, while the others died from esophageal cancer recurrence.

Specimens of esophageal cancer tissues were obtained from the enrolled patients with advanced ESCC who underwent surgical treatments with or without the preceding definitive CRT at Tohoku University Hospital. Furthermore, samples from patients with OSCCs were obtained from the those that underwent surgical treatments without any preceding neoadjuvant therapies at Tsurumi University Hospital or Kanto Rosai Hospital. This study was approved by the ethics committees of Tohoku University Graduate School of Medicine (permission number 2016-1-331), National Hospital Organization Sagamihara National Hospital (permission number 2018-051), Tsurumi University (permission number 1608), and Kanto Rosai Hospital (permission number 2018-25). Written informed consent was obtained prior to surgical treatment.

\subsection{RNA Extraction from Tumor Tissues}

Fresh specimens of ESCC tissues and OSCC tissues were obtained from all patients and immediately soaked in RNAlater Stabilization Solution (Invitrogen, Waltham, MA, USA) and / or eventually frozen at $-80^{\circ} \mathrm{C}$. Total RNA was extracted from each specimen using the RNeasy Lipid Tissue Mini Kit (Qiagen, Venio, the Netherlands) according to the manufacturer's instructions. Complementary DNA (cDNA) was synthesized from DNA-free RNA using the PrimeScript RT reagent Kit (Takara Bio, Tokyo, Japan) according to the manufacturer's instructions.

\subsection{T-Cell Receptor Repertoire Analysis}

TCR Repertoire analysis was performed using the Repertoire Analysis Kit according to the manufacturer's instructions (Repertoire Genesis, Ibaraki, Osaka, Japan). In brief, cDNA was synthesized using $h T R A$ or $h T R B$-specific reverse transcription primers, which were subsequently conjugated with universal adapters. The first PCR was performed by unbiased PCR by using a nested PCR primer set that amplified the $\mathrm{V}, \mathrm{J}$, and $\mathrm{C}$ regions of $h T R A$ and the $\mathrm{V}, \mathrm{D}, \mathrm{J}$, and $\mathrm{C}$ regions of $h T R B$. The first PCR products were confirmed by electrophoresis. The obtained PCR products were purified using Agencourt AMPure XP (Beckman Coulter, Brea, CA, USA). The concentration of the purified product was measured using a Qubit Fluorometer (Thermo Fisher Scientific, Waltham, MA, USA) and, finally, diluted to $4 \mathrm{nM}$. The final products were sequenced by Mi-Seq (Illumina, San Diego, CA, USA). The obtained sequence data were analyzed by TCR repertoire analysis software developed by Repertoire Genesis (Repertoire Genesis, Ibaraki, Osaka, Japan).

As a normal control, RNA was extracted from peripheral white blood cells from 18 healthy volunteers enrolled in this study under permission number 2015121708 of the ethics committee of the Clinical Research Center for Rheumatology and Allergy, National Hospital Organization Sagamihara National Hospital, as previously described [39]. TCR repertoire analyses were performed as described above.

\section{4. cDNA Synthesis and Quantitative Polymerase Chain Reaction}

The expression levels of immune response-related genes, including T-cell-related clusters of differentiation antigens and biomarkers, were measured by quantitative polymerase chain reaction (qPCR) using the Bio-Rad CFX96 system (Bio-Rad, Hercules, CA, USA). PCR primers for $C D 8 B$ and GAPDH were purchased from Takara Bio Inc. (Shiga, Japan), and the primers for $P D-L 1$ were obtained from a previous report [40]. The sequences of the primers were as follows: 
CD8B-forward 5'-GGCATCTACTTCTGCATGATCGTC-3'

CD8B-reverse 5'-TGGGTAACCGGCACACTCTC- $3^{\prime}$

PD-L1-forward 5'-AAATGGAACCTGGCGAAAGC-3'

PD-L1-reverse 5'-GATGAGCCCCTCAGGCATTT-3'

GAPDH-forward 5'-GCACCGTCAAGGCTGAGAAC- $3^{\prime}$

GAPDH-reverse 5'-ATGGTGGTGAAGACGCCAGT-3'.

cDNAs were synthesized from $500 \mathrm{ng}$ of total RNA isolated from each specimen of ESCC or OSCC using the Prime Script RT reagent Kit (Takara, Shiga, Japan) for each of the $10 \mathrm{qPCR}$ reactions. The PCR mixture consisted of $5 \mu \mathrm{L}$ of SsoFast EvaGreen Supermix (Bio-Rad, Hercules, CA, USA), $3.5 \mu \mathrm{L}$ of RNase/ DNase-free water, $0.5 \mu \mathrm{L}$ of $5-\mu \mathrm{M}$ primer mix, and $1-\mu \mathrm{L} c \mathrm{cNA}$ in a final total volume of $10 \mu \mathrm{L}$. This was then applied to the CFX96 system. Cycling conditions were as follows: $30 \mathrm{~s}$ at $95^{\circ} \mathrm{C}$, followed by 50 cycles of $1 \mathrm{~s}$ at $95{ }^{\circ} \mathrm{C}$ and $5 \mathrm{~s}$ at $60{ }^{\circ} \mathrm{C}$. At the end of each program, a melting curve analysis was performed from $70{ }^{\circ} \mathrm{C}$ to $94{ }^{\circ} \mathrm{C}$ to confirm homogeneity of the PCR products. All assays were performed in triplicate, and mean values were used to calculate the gene expression levels. For calibration of the absolute quantification, a standard curve for each target was prepared by five 10-fold serial dilutions of the standard nucleotide that was generated by the linearized plasmid cloned with the PCR product of each target. This was confirmed by DNA sequencing using the Sanger method and was quantified by SmartSpec3000 (Bio-Rad, Hercules, CA, USA).

\subsection{Diversity Metric Calculation}

The clonality index was defined as either Pielou's evenness, the Shannon-Weaver index $H$, or inverse Simpson's index $(1 / \lambda)$ and calculated as described previously [39,41].

\subsection{Statistical Analysis}

For continuous variables, the Shapiro-Wilk $W$ test for normality was performed for each group, at first. If the parametric conditions were fulfilled, an unpaired Student's $t$-test was performed, except for those that had statistical significance $(p<0.05)$ for the Levene's test, which were instead analyzed by Welch's $t$-test. For nonparametric conditions, the Mann-Whitney $\mathrm{U}$ test was performed. All analyses were performed using SPSS version 24 (IBM, Armonk, NY, USA). A bilateral $p$-value below 0.05 was considered statistically significant.

\section{Conclusions}

In this study, we showed a significant induction of oligoclonality of TIL in ESCC or OSCC when compared to healthy volunteers; further, we suggested the clonal expansion of TIL might be more notably induced when preceded by definitive CRT. Furthermore, our results suggested that a low ratio of $P D-L 1$ mRNA/CD $8 B$ mRNA in tumor tissues after definitive CRT could be a potential prognostic maker for salvage surgery, although the clonal expansion of TIL itself was not enough to improve the prognosis there. We predicted that not only the clonal expansion of TIL but, also, PD-L1 suppression, which is likely upregulated during chemotherapy and chemoradiation therapy, should play an important role in improving the prognosis. This viewpoint was supported by previous results in other types of cancers, such as extrahepatic bile duct cancers, non-small cell lung carcinoma, or tongue squamous cell carcinoma $[18,21,22,36,37]$. However, the results presented here should be validated by another clinical cohort because of the limited number of enrolled patients, especially for the prognostic study of salvage surgery after definitive CRT, and also validated by using endoscopic biopsies before salvage surgeries to establish the predictive markers.

Supplementary Materials: The following are available online at https:/ / www.mdpi.com/1422-006 7/22/3/1098/s1. Table S1: The data used in this study; the results from the TCR repertoire analyses and from the quantitative PCR. 
Author Contributions: Conceptualization, T.M.; methodology, T.M. and R.S.; software, R.S.; validation, T.M., K.K. (Kenichi Kumagai), and R.S.; formal analysis, T.M. and R.S.; investigation, K.N., T.Y., and K.K. (Koji Kuwano); resources, T.M., K.K. (Kenichi Kumagai), Y.H., and H.K.; data curation, T.M., K.K. (Kenichi Kumagai), and R.S.; writing—original draft preparation, T.M.; writing-review and editing, T.M., K.K. (Kenichi Kumagai), and R.S.; visualization, T.M., T.Y., and R.S.; supervision, T.M.; project administration, T.M. and K.K. (Kenichi Kumagai); and funding acquisition, T.M. and Y.H. All authors have read and agreed to the published version of the manuscript.

Funding: This work was supported by Grant-in-aid from the Ministry of Education, Culture, Sports, Science and Technology (MEXT), Japan, under grant number 16K10488, between 2016 and 2018, by JSPS KAKENHI, grant number JP18H03008, between 2018 and 2020, and by the research fund from Repertoire Genesis in 2019.

Institutional Review Board Statement: The study was conducted according to the guidelines of the Declaration of Helsinki and approved by the Ethics Committees of Tohoku University Graduate School of Medicine (permission number 2016-1-331), National Hospital Organization Sagamihara National Hospital (permission number 2018-051), Tsurumi University (permission number 1608), and Kanto Rosai Hospital (permission number 2018-25).

Informed Consent Statement: Written informed consent was obtained prior to surgical treatment.

Data Availability Statement: The data presented in this study are available on request from the corresponding author.

Acknowledgments: The authors are grateful to Kazutaka Kitaura (Repertoire Genesis) for his helpful advice.

Conflicts of Interest: T.M. received a personal fee from OncoTherapy Science in 2017 outside the submitted work. R.S. received a personal fee from Repertoire Genesis. The authors declare no conflict of interest.

\section{References}

1. Chen, W.; Zheng, R.; Zuo, T.; Zeng, H.; Zhang, S.; He, J. National cancer incidence and mortality in China, 2012. Chin. J. Cancer Res. 2016, 28, 1-11. [PubMed]

2. Global Burden of Disease Cancer, C.; Fitzmaurice, C.; Allen, C.; Barber, R.M.; Barregard, L.; Bhutta, Z.A.; Brenner, H.; Dicker, D.J.; Chimed-Orchir, O.; Dandona, R.; et al. Global, regional, and national cancer incidence, mortality, years of life lost, years lived with disability, and disability-adjusted life-years for 32 cancer groups, 1990 to 2015: A systematic analysis for the global burden of disease study. JAMA Oncol. 2017, 3, 524-548. [CrossRef] [PubMed]

3. Tachimori, Y.; Ozawa, S.; Numasaki, H.; Ishihara, R.; Matsubara, H.; Muro, K.; Oyama, T.; Toh, Y.; Udagawa, H.; Uno, T.; et al. Comprehensive registry of esophageal cancer in Japan, 2011. Esophagus 2018, 15, 127-152. [CrossRef] [PubMed]

4. Bollschweiler, E.; Holscher, A.H.; Schmidt, M.; Warnecke-Eberz, U. Neoadjuvant treatment for advanced esophageal cancer: Response assessment before surgery and how to predict response to chemoradiation before starting treatment. Chin. J. Cancer Res. 2015, 27, 221-230. [PubMed]

5. Haisley, K.R.; Hart, K.D.; Nabavizadeh, N.; Bensch, K.G.; Vaccaro, G.M.; Thomas, C.R., Jr.; Schipper, P.H.; Hunter, J.G.; Dolan, J.P. Neoadjuvant chemoradiotherapy with concurrent cisplatin/5-fluorouracil is associated with increased pathologic complete response and improved survival compared to carboplatin/paclitaxel in patients with locally advanced esophageal cancer. Dis. Esophagus Off. J. Int. Soc. Dis. Esophagus ISDE 2017, 30, 1-7. [CrossRef]

6. Shao, M.S.; Wong, A.T.; Schwartz, D.; Weiner, J.P.; Schreiber, D. Definitive or preoperative chemoradiation therapy for esophageal cancer: Patterns of care and survival outcomes. Ann. Thorac. Surg. 2016, 101, 2148-2154. [CrossRef]

7. Swisher, S.G.; Moughan, J.; Komaki, R.U.; Ajani, J.A.; Wu, T.T.; Hofstetter, W.L.; Konski, A.A.; Willett, C.G. Final results of NRG oncology RTOG 0246: An organ-preserving selective resection strategy in esophageal cancer patients treated with definitive chemoradiation. J. Thorac. Oncol. Off. Publ. Int. Assoc. Study Lung Cancer 2017, 12, 368-374. [CrossRef]

8. Rawat, S.; Kumar, G.; Kakria, A.; Sharma, M.K.; Chauhan, D. Chemoradiotherapy in the management of locally advanced squamous cell carcinoma esophagus: Is surgical resection required? J. Gastrointest. Cancer 2013, 44, 277-284. [CrossRef]

9. Teoh, A.Y.; Chiu, P.W.; Yeung, W.K.; Liu, S.Y.; Wong, S.K.; Ng, E.K. Long-term survival outcomes after definitive chemoradiation versus surgery in patients with resectable squamous carcinoma of the esophagus: Results from a randomized controlled trial. Ann. Oncol. Off. J. Eur. Soc. Med Oncol. ESMO 2013, 24, 165-171. [CrossRef]

10. Yoo, C.; Park, J.H.; Yoon, D.H.; Park, S.I.; Kim, H.R.; Kim, J.H.; Jung, H.Y.; Lee, G.H.; Choi, K.D.; Song, H.J.; et al. Salvage esophagectomy for locoregional failure after chemoradiotherapy in patients with advanced esophageal cancer. Ann. Thorac. Surg. 2012, 94, 1862-1868. [CrossRef] [PubMed] 
11. Wu, X.; Gu, J.; Wu, T.T.; Swisher, S.G.; Liao, Z.; Correa, A.M.; Liu, J.; Etzel, C.J.; Amos, C.I.; Huang, M.; et al. Genetic variations in radiation and chemotherapy drug action pathways predict clinical outcomes in esophageal cancer. J. Clin. Oncol. 2006, 24, 3789-3798. [CrossRef] [PubMed]

12. Adenis, A.; Tresch, E.; Dewas, S.; Romano, O.; Messager, M.; Amela, E.; Clisant, S.; Kramar, A.; Mariette, C.; Mirabel, X. Clinical complete responders to definite chemoradiation or radiation therapy for oesophageal cancer: Predictors of outcome. BMC Cancer 2013, 13, 413. [CrossRef] [PubMed]

13. Schneider, P.M.; Metzger, R.; Schaefer, H.; Baumgarten, F.; Vallbohmer, D.; Brabender, J.; Wolfgarten, E.; Bollschweiler, E.; Baldus, S.E.; Dienes, H.P.; et al. Response evaluation by endoscopy, rebiopsy, and endoscopic ultrasound does not accurately predict histopathologic regression after neoadjuvant chemoradiation for esophageal cancer. Ann. Surg. 2008, 248, 902-908. [CrossRef] [PubMed]

14. Park, J.H.; Jang, M.; Tarhan, Y.E.; Katagiri, T.; Sasa, M.; Miyoshi, Y.; Kalari, K.R.; Suman, V.J.; Weinshilboum, R.; Wang, L.; et al. Clonal expansion of antitumor $\mathrm{T}$ cells in breast cancer correlates with response to neoadjuvant chemotherapy. Int. J. Oncol. 2016, 49, 471-478. [CrossRef]

15. Dovedi, S.J.; Cheadle, E.J.; Popple, A.L.; Poon, E.; Morrow, M.; Stewart, R.; Yusko, E.C.; Sanders, C.M.; Vignali, M.; Emerson, R.O.; et al. Fractionated radiation therapy stimulates antitumor immunity mediated by both resident and infiltrating polyclonal T-cell Populations when combined with PD-1 blockade. Clin. Cancer Res. 2017, 23, 5514-5526. [CrossRef]

16. Zhang, C.; Palashati, H.; Tan, Q.; Ku, W.; Miao, Y.; Xiong, H.; Lu, Z. Immediate and substantial evolution of T-cell repertoire in peripheral blood and tumor microenvironment of patients with esophageal squamous cell carcinoma treated with preoperative chemotherapy. Carcinogenesis 2018, 39, 1389-1398. [CrossRef]

17. Chow, J.; Hoffend, N.C.; Abrams, S.I.; Schwaab, T.; Singh, A.K.; Muhitch, J.B. Radiation induces dynamic changes to the T cell repertoire in renal cell carcinoma patients. Proc. Natl. Acad. Sci. USA 2020, 117, 23721-23729. [CrossRef]

18. Lim, Y.J.; Koh, J.; Kim, K.; Chie, E.K.; Kim, B.; Lee, K.B.; Jang, J.Y.; Kim, S.W.; Oh, D.Y.; Bang, Y.J.; et al. High ratio of programmed cell death protein $1(\mathrm{PD}-1)(+) / C D 8(+)$ tumor-infiltrating lymphocytes identifies a poor prognostic subset of extrahepatic bile duct cancer undergoing surgery plus adjuvant chemoradiotherapy. Radiother. Oncol. 2015, 117, 165-170. [CrossRef]

19. Jiang, Y.; Lo, A.W.I.; Wong, A.; Chen, W.; Wang, Y.; Lin, L.; Xu, J. Prognostic significance of tumor-infiltrating immune cells and PD-L1 expression in esophageal squamous cell carcinoma. Oncotarget 2017, 8, 30175-30189. [CrossRef]

20. Fumet, J.D.; Richard, C.; Ledys, F.; Klopfenstein, Q.; Joubert, P.; Routy, B.; Truntzer, C.; Gagne, A.; Hamel, M.A.; Guimaraes, C.F.; et al. Prognostic and predictive role of CD8 and PD-L1 determination in lung tumor tissue of patients under anti-PD-1 therapy. Br. J. Cancer 2018, 119, 950-960. [CrossRef]

21. Yoneda, K.; Kuwata, T.; Kanayama, M.; Mori, M.; Kawanami, T.; Yatera, K.; Ohguri, T.; Hisaoka, M.; Nakayama, T.; Tanaka, F. Alteration in tumoural PD-L1 expression and stromal CD8-positive tumour-infiltrating lymphocytes after concurrent chemoradiotherapy for non-small cell lung cancer. Br. J. Cancer 2019, 121, 490-496. [CrossRef] [PubMed]

22. Gennen, K.; Kasmann, L.; Taugner, J.; Eze, C.; Karin, M.; Roengvoraphoj, O.; Neumann, J.; Tufman, A.; Orth, M.; Reu, S.; et al. Prognostic value of PD-L1 expression on tumor cells combined with CD8+ TIL density in patients with locally advanced non-small cell lung cancer treated with concurrent chemoradiotherapy. Radiat. Oncol. 2020, 15, 5. [CrossRef]

23. Qian, D.; Wang, Y.; Zhao, G.; Cao, F.; Er, P.; Chen, X.; Cheng, J.; Zhang, W.; Li, X.; Zhang, B.; et al. Tumor Remission and Tumor-Infiltrating Lymphocytes During Chemoradiation Therapy: Predictive and Prognostic Markers in Locally Advanced Esophageal Squamous Cell Carcinoma. Int. J. Radiat. Oncol. Biol. Phys. 2019, 105, 319-328. [CrossRef] [PubMed]

24. Ando, N.; Kato, H.; Igaki, H.; Shinoda, M.; Ozawa, S.; Shimizu, H.; Nakamura, T.; Yabusaki, H.; Aoyama, N.; Kurita, A.; et al. A randomized trial comparing postoperative adjuvant chemotherapy with cisplatin and 5-fluorouracil versus preoperative chemotherapy for localized advanced squamous cell carcinoma of the thoracic esophagus (JCOG9907). Ann. Surg. Oncol. 2012, 19, 68-74. [CrossRef] [PubMed]

25. Stahl, M.; Walz, M.K.; Stuschke, M.; Lehmann, N.; Meyer, H.J.; Riera-Knorrenschild, J.; Langer, P.; Engenhart-Cabillic, R.; Bitzer, M.; Konigsrainer, A.; et al. Phase III comparison of preoperative chemotherapy compared with chemoradiotherapy in patients with locally advanced adenocarcinoma of the esophagogastric junction. J. Clin. Oncol. 2009, 27, 851-856. [CrossRef]

26. Boffetta, P.; Hashibe, M. Alcohol and cancer. Lancet Oncol. 2006, 7, 149-156. [CrossRef]

27. Pelucchi, C.; Gallus, S.; Garavello, W.; Bosetti, C.; La Vecchia, C. Alcohol and tobacco use, and cancer risk for upper aerodigestive tract and liver. Eur. J. Cancer Prev. 2008, 17, 340-344. [CrossRef]

28. Khammanivong, A.; Anandharaj, A.; Qian, X.; Song, J.M.; Upadhyaya, P.; Balbo, S.; Bandyopadhyay, D.; Dickerson, E.B.; Hecht, S.S.; Kassie, F. Transcriptome profiling in oral cavity and esophagus tissues from (S)-N'-nitrosonornicotine-treated rats reveals candidate genes involved in human oral cavity and esophageal carcinogenesis. Mol. Carcinog. 2016, 55, $2168-2182$. [CrossRef]

29. Liu, H.; Zhang, B.; Sun, Z. Spectrum of EGFR aberrations and potential clinical implications: Insights from integrative pan-cancer analysis. Cancer Commun. 2020, 40, 43-59. [CrossRef]

30. Chen, D.S.; Mellman, I. Oncology meets immunology: The cancer-immunity cycle. Immunity 2013, 39, 1-10. [CrossRef]

31. Bernal-Estevez, D.; Sanchez, R.; Tejada, R.E.; Parra-Lopez, C. Chemotherapy and radiation therapy elicits tumor specific T cell responses in a breast cancer patient. BMC Cancer 2016, 16, 591. [CrossRef] [PubMed] 
32. Sanz-Pamplona, R.; Melas, M.; Maoz, A.; Schmit, S.L.; Rennert, H.; Lejbkowicz, F.; Greenson, J.K.; Sanjuan, X.; Lopez-Zambrano, M.; Alonso, M.H.; et al. Lymphocytic infiltration in stage II microsatellite stable colorectal tumors: A retrospective prognosis biomarker analysis. PLoS Med. 2020, 17, e1003292. [CrossRef] [PubMed]

33. Fukuoka, E.; Yamashita, K.; Tanaka, T.; Sawada, R.; Sugita, Y.; Arimoto, A.; Fujita, M.; Takiguchi, G.; Matsuda, T.; Oshikiri, T.; et al. Neoadjuvant Chemotherapy Increases PD-L1 Expression and CD8(+) Tumor-infiltrating Lymphocytes in Esophageal Squamous Cell Carcinoma. Anticancer Res. 2019, 39, 4539-4548. [CrossRef] [PubMed]

34. Keane, C.; Gould, C.; Jones, K.; Hamm, D.; Talaulikar, D.; Ellis, J.; Vari, F.; Birch, S.; Han, E.; Wood, P.; et al. The T-cell receptor repertoire influences the tumor microenvironment and is associated with survival in aggressive B-cell lymphoma. Clin. Cancer Res. 2017, 23, 1820-1828. [CrossRef] [PubMed]

35. Schumacher, K.; Haensch, W.; Roefzaad, C.; Schlag, P.M. Prognostic significance of activated CD8(+) T cell infiltrations within esophageal carcinomas. Cancer Res. 2001, 61, 3932-3936.

36. Tokito, T.; Azuma, K.; Kawahara, A.; Ishii, H.; Yamada, K.; Matsuo, N.; Kinoshita, T.; Mizukami, N.; Ono, H.; Kage, M.; et al. Predictive relevance of PD-L1 expression combined with CD8+ TIL density in stage III non-small cell lung cancer patients receiving concurrent chemoradiotherapy. Eur. J. Cancer 2016, 55, 7-14. [CrossRef]

37. Troiano, G.; Rubini, C.; Togni, L.; Caponio, V.C.A.; Zhurakivska, K.; Santarelli, A.; Cirillo, N.; Lo Muzio, L.; Mascitti, M. The immune phenotype of tongue squamous cell carcinoma predicts early relapse and poor prognosis. Cancer Med. 2020, 9, 8333-8344. [CrossRef]

38. Chen, Y.; Lu, Y.; Wang, Y.; Yang, H.; Xia, Y.; Chen, M.; Song, H.; Li, T.; Li, D.; Wang, J.; et al. Comparison of salvage chemoradiation versus salvage surgery for recurrent esophageal squamous cell carcinoma after definitive radiochemotherapy or radiotherapy alone. Dis. Esophagus Off. J. Int. Soc. Dis. Esophagus ISDE 2014, 27, 134-140. [CrossRef]

39. Kitaura, K.; Shini, T.; Matsutani, T.; Suzuki, R. A new high-throughput sequencing method for determining diversity and similarity of $\mathrm{T}$ cell receptor (TCR) alpha and beta repertoires and identifying potential new invariant TCR alpha chains. BMC Immunol. 2016, 17, 38. [CrossRef]

40. Hassan, S.S.; Akram, M.; King, E.C.; Dockrell, H.M.; Cliff, J.M. PD-1, PD-L1 and PD-L2 gene expression on T-cells and natural killer cells declines in conjunction with a reduction in PD-1 protein during the intensive phase of tuberculosis treatment. PLoS ONE 2015, 10, e0137646. [CrossRef]

41. Carey, A.J.; Hope, J.L.; Mueller, Y.M.; Fike, A.J.; Kumova, O.K.; van Zessen, D.B.H.; Steegers, E.A.P.; van der Burg, M.; Katsikis, P.D. Public clonotypes and convergent recombination characterize the naive CD8(+) T-cell receptor repertoire of extremely preterm neonates. Front. Immunol. 2017, 8, 1859. [CrossRef] [PubMed] 